\title{
¿Son efectivas las intervenciones para reducir el consumo de sodio en la dieta?
}

\author{
Gabriel Rada ${ }^{1,2^{*}}$
}

Resumen - Las personas que consumen más sodio tienen cifras de presión arterial más alta, lo cual aumenta su morbimortalidad cardiovascular. El consumo de sodio en países latinoamericanos duplica o triplica lo recomendado, lo que ha llevado a redoblar los esfuerzos por abordar este problema. Los cambios de conducta y modificación de estilos de vida son difíciles de conseguir y mantener en el tiempo, por lo que las intervenciones a este nivel suelen precisar un abordaje desde múltiples perspectivas.

Palabras Clave: Sodio; presión arterial; intervención

Abstract - People who eat more sodium have higher blood pressure values, which increases their cardiovascular morbidity and mortality. Sodium intake in Latin American countries doubled or tripled the recommended consumption, which has led to increase efforts to address this problem. Changes in behavior and lifestyle modification are difficult to achieve and maintain over time, because of this interventions at this level typically require an approach from multiple perspectives.

Keywords: Sodium, blood pressure, intervention

Fecha de envío: 23 de Mayo de 2012 - Fecha de aceptación: 5 de 0ctubre de 2012

\section{Este resumen se basa en la siguiente revisión sistemática:}

Taylor RS, Ashton KE, Moxham T, Hooper L, Ebrahim S. Reduced dietary salt for the prevention of cardiovascular disease. Cochrane Database of Systematic Reviews 2011, Issue 7. Art. No.: CD009217.

\section{Mensajes clave}

- En pacientes normotensos e hipertensos las intervenciones para reducir el consumo de sal en la dieta podrían disminuir la morbilidad cardiovascular.

- La escasa reducción en el consumo de sodio que se logra con las intervenciones para reducir la sal en la dieta podría no ser suficiente para disminuir la mortalidad en normotensos o hipertensos.

- En pacientes con insuficiencia cardiaca no es posible saber si la reducción de sodio disminuye la mortalidad, debido a que la calidad de la evidencia es muy baja, y no se encontraron estudios que reportaran el impacto sobre la morbilidad cardiovascular.

\section{¿Para quién es este resumen?}

Personal médico, de enfermería o nutrición que atiende a población general o hipertensa, o que toma decisiones respecto a implementar programas para la reducción del consumo de sodio.

\section{Este resumen incluye:}

- Hallazgos clave de investigaciones basadas en una revisión sistemática

- Consideraciones sobre la relevancia de esta investigación para países de medianos y bajos ingresos

No Incluye:

- Recomendaciones

- Evidencia adicional no incluída en la revisión sistemática - Descripciones detalladas de las intervenciones o su implementación

(1) Programa de Salud Basado en Evidencias, Facultad de Medicina, Pontificia Universidad Católica de Chile, Santiago, Chile. (2)Hospital Dr. Sótero del Río, Puente Alto, Chile.

*Autor de correspondencia: gabriel@rada.cl 


\section{Antecedentes}

La Unidad de Medicina Basada en Evidencia selecciona aquellas revisiones que puedan ser de mayor interés para los profesionales de salud en Latinoamérica. Podría ser que una revisión de buena calidad no encuentre ningún estudio de buen diseño, o ninguno en países de bajos y medianos ingreso, o que sencillamente no encuentre ningún estudio. Aunque esto sea decepcionante, conocer qué es lo que no se sabe es tan importante como conocer qué es lo que se sabe. Se estima que anualmente se producen 17 millones de muertes por causa cardiovascular en el mundo. La hipertensión arterial es uno de los factores de riesgo importantes para el desarrollo de estas. El consumo de sodio produce una elevación de la presión arterial en el corto plazo, sin embargo para tener efectos a largo plazo y lograr un impacto en la prevención de accidentes cerebrovasculares, Infartos miocárdicos, y otros eventos cardiovasculares, se requiere que las personas mantengan un consumo reducido de sodio. Estudios y revisiones sistemáticas previos no han mostrado claramente que las intervenciones para reducir la ingesta de sal en la dieta se traduzcan en un cambio de conducta permanente en el tiempo, y por tanto, en una disminución de estos eventos.

\section{Acerca de la revisión sistemática que respalda este resumen}

Objetivo de la revisión: 1. Evaluar los efectos a largo plazo sobre la mortalidad y morbilidad cardiovascular de las intervenciones orientadas a reducir el consumo de sal en la dieta. 2. Investigar si la reducción de presión arterial es un factor explicatorio del efecto de estas intervenciones dietéticas sobre la morbimortalidad cardiovascular.(Taylor et al., 2011)

\begin{tabular}{|c|c|c|}
\hline & Qué buscaron los autores de la revisión & Qué encontraron los autores de la revisión \\
\hline Intervenciones & $\begin{array}{l}\text { Estudios controlados con asignación aleatoria (ECAs) } \\
\text { con seguimiento de al menos } 6 \text { meses, que evalua- } \\
\text { ran la reducción de ingesta de sal, comparada con } \\
\text { placebo, manejo estándar o no intervención. } \\
\text { Los estudios podían considerar el consejo para re- } \\
\text { ducir el consumo, o intervenciones específicas que } \\
\text { conllevaran una menor ingesta. } \\
\text { Para mortalidad a largo plazo, se consideraron estu- } \\
\text { dios observacionales de seguimiento posterior a la } \\
\text { finalización del estudio (post-randomization follow up). }\end{array}$ & $\begin{array}{l}7 \text { ECAs. } \\
4 \text { estudios consistían en programas de intervención dietética integral } \\
\text { y cambio de conducta, liderados por personal especializado. Incluye- } \\
\text { ron consejería grupal regular, boletines, autoevaluaciones, estableci- } \\
\text { miento de metas, degustación de comida y recetas de cocina. } \\
1 \text { estudio comunitario (cluster } R C T \text { ) de un programa dietético, en que } \\
\text { los pacientes fueron asignados a distintas cocinas, en las cuales se uti- } \\
\text { lizaba sal potásica o sal habitual. } \\
1 \text { estudio de solo consejería, repetida a los } 6 \text { meses. } \\
1 \text { estudio de instructivos escritos con una dieta de } 5 \text { gramos de sodio, } \\
\text { preparada por nutricionistas. }\end{array}$ \\
\hline Participantes & $\begin{array}{l}\text { Adultos (>18 años). Se excluyeron estudios en muje- } \\
\text { res embarazadas y en niños. }\end{array}$ & $\begin{array}{l}\text { Hipertensos (2), normotensos (3), mezcla de hipertensos y normoten- } \\
\text { sos (1), insuficiencia cardiaca (1). }\end{array}$ \\
\hline Lugares & Sin restricciones. & Estados Unidos de América (4), Australia (1), Italia (1) y Taiwán (1). \\
\hline Resultados & $\begin{array}{l}\text { Mortalidad, morbilidad cardiovascular (infarto mio- } \\
\text { cárdico, accidente cerebrovascular, angina, insu- } \\
\text { ficiencia cardiaca, eventos vasculares periféricos, } \\
\text { muerte súbita, revascularización, hospitalizaciones). } \\
\text { En los estudios que midieron alguno de los ante- } \\
\text { riores, también se buscó presión arterial, excreción } \\
\text { urinaria de sodio (u otro método de estimación de } \\
\text { ingesta), y calidad de vida. }\end{array}$ & $\begin{array}{l}\text { Mortalidad por cualquier causa (5), morbilidad cardiovascular (4). } \\
\text { Todos los estudios reportaron adherencia, excreción urinaria de sodio } \\
\text { y presión arterial. } \\
\text { Ningún estudio reportó adecuadamente calidad de vida. }\end{array}$ \\
\hline \multicolumn{3}{|c|}{ Fecha de la búsqueda más reciente: octubre 2008.} \\
\hline
\end{tabular}




\section{Resumen de lo encontrado por la revisión}

Se identificaron 7 estudios con medición de morbilidad cardiovascular o de mortalidad en el largo plazo ( $>6$ meses). Se encontraron estudios en hipertensos y normotensos, y en pacientes con insuficiencia cardiaca. Los pacientes fueron bastante diversos en términos de género y raza. La mayoría de los estudio evaluó intervenciones complejas, multidisciplinarias, y con múltiples componentes. Todos los estudios se llevaron a cabo en países de altos ingresos.

\section{Sobre la calidad de la evidencia (GRADE)}

La calidad de la evidencia es una opinión sobre el alcance hasta el cual podemos tener certeza de que las estimaciones de efectos sean correctas. Estas opiniones son emitidas utilizando el sistema GRADE y son brindadas para cada resultado. Los juicios se basan en el tipo de diseño del estudio (estudios clínicos aleatorizados versus estudios observacinales), el riesgo de sesgo, la coherencia de los resultados entre los estudios y la precisión del conjunto de estimaciones entre los estudios. Para cada resultado, la calidad de la evidencia se clasifica como alta, moderada, baja o muy baja. Para más información sobre GRADE: http://www.gradeworkinggroup.org/
Alta: Es poco probable que otras investigaciones cambien nuestra confianza en estos resultados acumulativos.

\section{0}

Moderada: Es posible que otras investigaciones tengan un impacto importante en nuestra confianza en el resultado acumulativo y es probable que este cambie.

\section{0}

Baja: Es muy probable que otras investigaciones tengan un impacto importante en nuestra confianza en el resultado acumulativo y es probable que este cambie.

0000

Muy baja: No podemos estar seguros sobre la estimación existente

\section{Efectos de las intervenciones para reducir el consumo de sodio dietético en pacientes normotensos}

- Las intervenciones para reducir el consumo de sodio en la dieta podrían disminuir la morbilidad cardiovascular en normotensos. La calidad de la evidencia es baja.

- En normotensos, no es posible saber si las intervenciones para reducir el consumo de sodio en la dieta disminuyen la mortalidad, debido a que la calidad de la evidencia es muy baja.

\begin{tabular}{|c|c|c|c|c|c|c|}
\hline \multicolumn{7}{|c|}{ Intervenciones par reducir el consumo de sodio y morbimortalidad cardiovascular en normotensos } \\
\hline \multicolumn{7}{|c|}{$\begin{array}{l}\text { Pacientes o población: Adultos con presión arterial normal } \\
\text { Lugar: Cualquiera } \\
\text { Intervención: Reducción de sodio en la dieta } \\
\text { Comparación: Manejo estándar, o no tratamiento }\end{array}$} \\
\hline \multirow[b]{2}{*}{$\begin{array}{l}\text { Desenlaces } \\
\text { (Outcomes) }\end{array}$} & \multicolumn{2}{|c|}{ Riesgos comparativos* } & \multirow[b]{2}{*}{$\begin{array}{l}\text { Riesgo } \\
\text { relativo } \\
(95 \% \mathrm{IC})\end{array}$} & \multirow[b]{2}{*}{$\begin{array}{l}\text { Número de } \\
\text { participantes } \\
\text { (estudios) }\end{array}$} & \multirow[b]{2}{*}{$\begin{array}{l}\text { Calidad de la } \\
\text { Evidencia (GRA- } \\
\text { DE) }\end{array}$} & \multirow[b]{2}{*}{ Comentarios } \\
\hline & $\begin{array}{l}\text { Sin restricción } \\
\text { de sodio }\end{array}$ & $\begin{array}{l}\text { Con restricción } \\
\text { de sodio }\end{array}$ & & & & \\
\hline Mortalidad & 21 por 1000 & 14 por 1000 (8 a 23) & $\begin{array}{l}0,67 \\
(0,40 \text { a } 1,12)\end{array}$ & $\begin{array}{l}3518 \\
(3)\end{array}$ & $\begin{array}{l}\text { OOO } \\
\text { Muy baja }\end{array}$ & $\begin{array}{l}\text { La calidad de la evidencia es } \\
\text { muy baja debido a la impreci- } \\
\text { sión del resultado y a algunas } \\
\text { limitaciones en la calidad de } \\
\text { los estudios. }\end{array}$ \\
\hline $\begin{array}{l}\text { Eventos } \\
\text { cardiovasculares }\end{array}$ & 90 por 1000 & $\begin{array}{l}64 \text { por } 1000 \\
(38 \text { a } 108)\end{array}$ & $\begin{array}{l}0,71 \\
(0,42 \text { a } 1,20)\end{array}$ & $\begin{array}{l}2505 \\
(2)\end{array}$ & $\begin{array}{l}\text { OOja } \\
\text { Baja }\end{array}$ & $\begin{array}{l}\text { La calidad de la evidencia es } \\
\text { baja debido a la imprecisión } \\
\text { del resultado (metaanálisis } \\
\text { combinando hipertensos y } \\
\text { normotensos aumenta la pre- } \\
\text { cisión**) y algunas limitaciones } \\
\text { en la calidad de los estudios. }\end{array}$ \\
\hline \multicolumn{7}{|c|}{$\begin{array}{l}\text { IC: Intervalo de confianza RR: Riesgo relativo GRADE: GRADE Working Group grades of evidence. } \\
\text { *Riesgos comparativos. Los riesgos que tiene una población que no recibe la intervención ("Sin restricción de sodio") están basados en los datos } \\
\text { de los estudios incluidos en la revisión sistemática. El riesgo correspondiente al grupo que recibe la intervención ("Con restricción de sodio") (y su } \\
\text { intervalo de confianza de } 95 \% \text { ) están basados en el riesgo relativo (y su intervalo de confianza de 95\%). } \\
\text { ** Reportado en He and MacGregor (2011) }\end{array}$} \\
\hline
\end{tabular}


Muy baja: No podemos estar seguros sobre la estimación existente.

\section{Efectos de las intervenciones para reducir el consumo de} sodio dietético en pacientes hipertensos

- Las intervenciones para reducir el consumo de sodio dietético podrían disminuir la morbilidad cardiovascular. La calidad de la evidencia es baja.
- Las intervenciones para reducir el consumo de sodio en la dieta podrían no conducir a diferencias en la mortalidad. La calidad de la evidencia es baja.

\begin{tabular}{|c|c|c|c|c|c|c|}
\hline \multicolumn{7}{|c|}{$\begin{array}{l}\text { Pacientes o población: Adultos con presión arterial elevada } \\
\text { Lugar: Cualquiera } \\
\text { Intervención: Reducción de sodio en la dieta } \\
\text { Comparación: Manejo estándar, o no tratamiento }\end{array}$} \\
\hline \multirow{2}{*}{$\begin{array}{l}\text { Desenlaces } \\
\text { (Outcomes) }\end{array}$} & \multicolumn{2}{|c|}{ Riesgos comparativos* } & \multirow{2}{*}{$\begin{array}{l}\text { Riesgo } \\
\text { relativo } \\
(95 \% \mathrm{IC})\end{array}$} & \multirow{2}{*}{$\begin{array}{l}\text { Número de } \\
\text { participantes } \\
\text { (estudios) }\end{array}$} & \multirow{2}{*}{$\begin{array}{l}\text { Calidad de } \\
\text { la Evidencia } \\
\text { (GRADE) }\end{array}$} & \multirow[b]{2}{*}{ Comentarios } \\
\hline & $\begin{array}{l}\text { Sin restricción } \\
\text { de sodio }\end{array}$ & $\begin{array}{l}\text { Con restricción } \\
\text { de sodio }\end{array}$ & & & & \\
\hline Mortalidad & 253 por 1000 & $\begin{array}{l}245 \text { por } 1000 \\
(210 \text { a } 285)\end{array}$ & $\begin{array}{l}\text { RR } 0,97 \\
(0,83 \text { a } 1,13)\end{array}$ & $\begin{array}{l}2058 \\
(2)\end{array}$ & $\begin{array}{l}\text { OOO } \\
\text { Baja }\end{array}$ & $\begin{array}{l}\text { La calidad de la evidencia } \\
\text { es muy baja debido a la im- } \\
\text { precisión del resultado y a } \\
\text { algunas limitaciones en la } \\
\text { calidad de los estudios. }\end{array}$ \\
\hline $\begin{array}{l}\text { Morbilidad } \\
\text { cardiovascular }\end{array}$ & 140 por 1000 & $\begin{array}{l}118 \text { por } 1000 \\
(80 \text { a } 172)\end{array}$ & $\begin{array}{l}0,84 \\
(0,57 \text { a } 1,24)\end{array}$ & $\begin{array}{l}720 \\
(2)\end{array}$ & $\begin{array}{l}000 \\
\text { Baja }\end{array}$ & $\begin{array}{l}\text { La calidad de la evidencia } \\
\text { es baja debido a la impre- } \\
\text { cisión del resultado (me- } \\
\text { taanálisis combinando hi- } \\
\text { pertensos y normotensos } \\
\text { aumenta la precisión }{ }^{* *} \text { ) y } \\
\text { a algunas limitaciones en } \\
\text { la calidad de los estudios. }\end{array}$ \\
\hline \multicolumn{7}{|c|}{$\begin{array}{l}\text { IC: Intervalo de confianza RR: Riesgo relativo GRADE: GRADE Working Group grades of evidence (ver la tercera y última página). } \\
\text { *Riesgos comparativos. Los riesgos que tiene una población que no recibe la intervención ("Sin restricción de sodio") están basados en los datos } \\
\text { de los estudios incluidos en la revisión sistemática. El riesgo correspondiente al grupo que recibe la intervención ("Con restricción de sodio") (y su } \\
\text { intervalo de confianza de 95\%) están basados en el riesgo relativo (y su intervalo de confianza de 95\%). }\end{array}$} \\
\hline
\end{tabular}




\section{Efectos de la reducción de sodio sobre la morbimortalidad cardiovascular en pacientes con insuficiencia cardiaca}

- No se encontraron estudios que reportaran el impacto de las intervenciones para reducir el consumo de sodio en la morbilidad cardiovascular.
- No es posible saber si las intervenciones para reducir el consumo de sal en la dieta disminuyen la mortalidad, debido a que la calidad de la evidencia es muy baja.

\begin{tabular}{|c|c|c|c|c|c|c|}
\hline \multicolumn{7}{|c|}{ Intervenciones par reducir el consumo de sodio y morbimortalidad cardiovascular en insuficiencia cardiaca } \\
\hline \multicolumn{7}{|c|}{ Pacientes o población: Adultos con insuficiencia cardiaca } \\
\hline \multicolumn{7}{|l|}{ Lugar: Cualquiera } \\
\hline \multicolumn{7}{|c|}{ Intervención: Reducción de sodio en la dieta } \\
\hline \multicolumn{7}{|c|}{ Comparación: Manejo estándar, o no tratamiento } \\
\hline \multirow[b]{2}{*}{ Outcomes } & \multicolumn{2}{|c|}{ Comparative risks* } & \multirow[b]{2}{*}{$\begin{array}{l}\text { Relative } \\
\text { effect } \\
(95 \% \mathrm{Cl})\end{array}$} & \multirow[b]{2}{*}{$\begin{array}{l}\text { Number of } \\
\text { participants } \\
\text { (studies) }\end{array}$} & \multirow[b]{2}{*}{$\begin{array}{l}\text { Quality of } \\
\text { the evidence } \\
\text { (GRADE) }\end{array}$} & \multirow[b]{2}{*}{ Comments } \\
\hline & $\begin{array}{l}\text { Sin restricción } \\
\text { de sodio }\end{array}$ & $\begin{array}{l}\text { Con restricción } \\
\text { de sodio }\end{array}$ & & & & \\
\hline Mortalidad & 51 por 1000 & $\begin{array}{l}132 \text { por } 1000 \\
(53 \text { a } 327)\end{array}$ & $\begin{array}{l}2,59 \\
(1,04 \text { a } 6,44)\end{array}$ & $\begin{array}{l}232 \\
(1)\end{array}$ & $\begin{array}{l}\text { OOOO } \\
\text { Muy baja }\end{array}$ & $\begin{array}{l}\text { La calidad de la evi- } \\
\text { dencia es muy baja } \\
\text { debido a limitaciones } \\
\text { metodológicas, y a } \\
\text { las marcadas diferen- } \\
\text { cias con el manejo } \\
\text { habitual de la insuf- } \\
\text { ciencia cardiaca en el } \\
\text { único estudio encon- } \\
\text { trado. }\end{array}$ \\
\hline $\begin{array}{l}\text { Morbilidad } \\
\text { cardiovascular }\end{array}$ & $\begin{array}{l}\text { No se encontra } \\
\text { el impacto de I } \\
\text { morbilidad car }\end{array}$ & $\begin{array}{l}\text { udios que evaluaran } \\
\text { ción de sodio en la } \\
\text { ular. }\end{array}$ & ---------- & $\begin{array}{l}0 \\
(0)\end{array}$ & ---------- & ----------------------- \\
\hline \multicolumn{7}{|c|}{$\begin{array}{l}\text { IC: Intervalo de confianza RR: Riesgo relativo GRADE: GRADE Working Group grades of evidence. } \\
\text { *Riesgos comparativos. Los riesgos que tiene una población que no recibe la intervención ("Sin restricción de sodio") están basados en los datos } \\
\text { de los estudios incluidos en la revisión sistemática. El riesgo correspondiente al grupo que recibe la intervención ("Con restricción de sodio") (y } \\
\text { su intervalo de confianza de 95\%) están basados en el riesgo relativo (y su intervalo de confianza de 95\%). }\end{array}$} \\
\hline
\end{tabular}


Relevancia de la revisión para países de medianos y bajos ingresos

\begin{tabular}{|c|c|}
\hline$\Rightarrow$ Hallazgos & $D$ Interpretación* \\
\hline \multicolumn{2}{|l|}{ APLICABILIDAD } \\
\hline $\begin{array}{l}\Rightarrow \text { Las intervenciones para reducir el consumo de sodio } \\
\text { podrían disminuir la morbilidad en normotensos e hi- } \\
\text { pertensos. El efecto en la mortalidad es difícil de preci- } \\
\text { sar debido a la baja o muy baja calidad de la evidencia. } \\
\Rightarrow \text { En pacientes con insuficiencia cardiaca no es posible } \\
\text { saber el efecto sobre la mortalidad o morbilidad, debi- } \\
\text { do a la muy baja calidad de la evidencia para la primera, } \\
\text { y a la ausencia de información para la última. } \\
\Rightarrow \text { La adherencia a la intervención fue baja en los es- } \\
\text { tudios, al igual que la disminución en la excreción de } \\
\text { sodio y el descenso de la presión arterial. } \\
\Rightarrow \text { Todos los estudios incluídos fueron hechos en paí- } \\
\text { ses de altos ingresos. }\end{array}$ & $\begin{array}{l}D \text { Considerando la dirección del efecto sobre la morbilidad, el conocimiento } \\
\text { fisiopatológico previo, y la abundante evidencia proveniente de estudios obser- } \\
\text { vacionales, esta revisión sugiere que la evidencia proveniente de ECAs acerca de } \\
\text { las intervenciones para reducir el consumo de sodio es muy limitada, y no que } \\
\text { la restricción de sodio no tenga efecto, como se ha planteado en comentarios y } \\
\text { notas de prensa. } \\
D \text { A pesar de tratarse de intervenciones complejas y con múltiples componentes, } \\
\text { el efecto sobre la presión arterial y sobre la excreción de sodio fue modesto, lo } \\
\text { cual explica en buena parte los resultados. } \\
D \text { El aumento de la mortalidad en el estudio de restricción de sodio en pacientes } \\
\text { con insuficiencia cardiaca constituye evidencia de muy baja calidad, sin embargo } \\
\text { pone una nota de cautela, y pone de manifiesto la necesidad de nuevos estudios. }\end{array}$ \\
\hline \multicolumn{2}{|l|}{ EQUIDAD } \\
\hline $\begin{array}{l}\Rightarrow \text { Esta revisión no reportó aspectos relacionados con } \\
\text { equidad }\end{array}$ &  \\
\hline \multicolumn{2}{|l|}{ CONSIDERACIONES ECONÓMICAS } \\
\hline $\begin{array}{l}\rightarrow \text { Esta revisión no reportó costos ni otras conside- } \\
\text { raciones económicas, sin embargo, la mayoría de los } \\
\text { estudios evaluaron intervenciones que conllevan un } \\
\text { alto costo en recursos humanos, o directamente en } \\
\text { alimentos o sal potásica. }\end{array}$ & $\begin{array}{l}D \text { Basándose en la información existente es difícil calcular el costo- beneficio de } \\
\text { estas intervenciones. Sin embargo, los efectos observados en esta revisión sugie- } \\
\text { ren que es altamente improbable que las intervenciones de alta complejidad o } \\
\text { de alto costo sean costo-efectivas. } \\
D \text { El desafío para los clínicos y los encargados de la salud pública es encontrar } \\
\text { intervenciones efectivas para reducir el consumo que sean factibles y de bajo } \\
\text { costo. }\end{array}$ \\
\hline \multicolumn{2}{|l|}{ MONITOREO Y EVALUACIÓN } \\
\hline $\begin{array}{l}\text { Llama la atención el reducido número de estudios } \\
\text { midiendo desenlaces importantes para el paciente y } \\
\text { de largo plazo. } \\
\Rightarrow \text { Ningún estudio reportó calidad de vida. }\end{array}$ & $\begin{array}{l}D \text { Futuros estudios deben enfocarse en la medición de mortalidad y morbilidad } \\
\text { cardiovascular a largo plazo. } \\
D \text { Considerando que las intervenciones requieren una modificación sustancial } \\
\text { del estilo de vida, es deseable que futuros estudios midan calidad de vida. }\end{array}$ \\
\hline
\end{tabular}

*Opiniones hechas por los autores de este resumen, y no necesariamente por los autores de la revisión. Están basadas en los hallazgos de la revisión y en literatura relacionada. Para más detalles acerca de cómo se elaboran estas opiniones ver: http://supportsummaries.org/support-summaries/how-support-summaries-are-prepared/ 


\section{Agradecimientos:}

A Joaquín Montero, Ignacio Neumann y Tomás Pantoja, por su acuciosa revisión y comentarios. Colaboradores SUPPORT: The Alliance for Health Policy and Systems Research (HPSR) es una colaboración internacional cuyo objetivo es promover la generación y el uso de investigación en políticas y sistemas de salud como medio para mejorar los sistemas de salud de los países en vías de desarrollo. www.who.int/alliance-hpsr. The Cochrane Effective Practice and Organisation of Care Group (EPOC) es un Grupo Revisor Colaborativo de la Colaboración Cochrane: una organización internacional cuyo objetivo es ayudar a que las personas tomen decisiones bien fundadas sobre la atención de salud perparando, manteniendo y asegurando el acceso a revisiones sistemáticas sobre los efectos de las intervenciones. www.epoc. cochrane.org. The Evidence-Informed Policy Network (EVIPNet) es una iniciativa para promover el uso de la investigación en salud en la toma de decisiones. Enfocándose en países de medianos y bajos ingresos. EVIPNet promueve la colaboración entre los responsables de la toma de decisión, los investigadores y la sociedad civil de un país, con el fin de facilitar tanto el desarrollo como la implementación de políticas a través del uso de la mejor evidencia científica disponible. www.who.int/rpc/evipnet/en/. Para más información, visite: www.supportsummaries.org. Ningún conflict de interés fue declarado para más detalles ver: http://supportsummaries.org/support-summaries/conflicts-of-interest/

\section{Literatura relacionada}

He FJ \& MacGregor GA. (2011). Salt reduction lowers cardiovascular risk: meta-analysis of outcome trials. Lancet $\mathbf{3 7 8}$, 380-382.

Matyas E, Jeitler K, Horvath K, Semlitsch T, Hemkens LG, Pignitter N \& Siebenhofer A. (2011). Benefit assessment of salt reduction in patients with hypertension: systematic overview. Journal of hypertension 29, 821-828.

Organización Panamericana de la Salud. Declaración Política sobre la Prevención de las enfermedades cardiovasculares mediante la reducción de la ingesta de sal alimentaria de toda la población Consultado el: (13 Enero de 2012). http://new.paho.org/hq/index.php?option=com content\&task=view\&id=1769\&ltemid=1\&lang=es

Support Summaries. How SUPPORT Summaries are prepared. Consultado el: (13 de enero de 2012). http://www. supportsummaries.org/support-summaries/how-supportsummaries-are-prepared/

Taylor RS, Ashton KE, Moxham T, Hooper L \& Ebrahim S. (2011). Reduced dietary salt for the prevention of cardiovascular disease. The Cochrane database of systematic reviews, CD009217. 